\title{
Canadian consensus: a new systemic treatment algorithm for advanced EGFR-mutated non-small-cell lung cancer
}

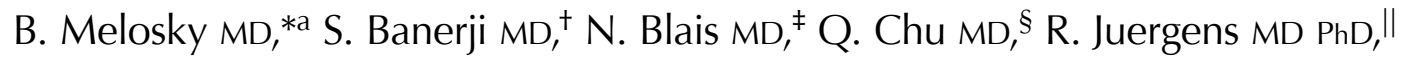 \\ N.B. Leighl MD MMSc, ${ }^{\#}$ G. Liu MD, ${ }^{\#}$ and P. Cheema MD**a
}

\begin{abstract}
Background Multiple clinical trials for the treatment of advanced EGFR-mutated non-small-cell lung cancer (NSCLC) have recently been reported. As a result, the treatment algorithm has changed, and many important clinical questions have been raised:

What is the optimal first-line treatment for patients with EGFR-mutated NSCLC?

What is preferred first-line treatment for patients with brain metastasis?

What is the preferred second-line treatment for patients who received first-line first- or second-generation tyrosine kinase inhibitors (TKIs)?

What is the preferred treatment after osimertinib?

What evidence do we have for treating patients whose tumours harbour uncommon EGFR mutations?
\end{abstract}

Methods A Canadian expert panel was convened to define the key clinical questions, review recent evidence, and discuss and agree on practice recommendations for the treatment of advanced EGFR-mutated NSCLC.

Results The published overall survival results for osimertinib, combined with its central nervous system activity, have led to osimertinib becoming the preferred first-line treatment for patients with common EGFR mutations, including those with brain metastasis. Other agents could still have a role, especially when osimertinib is not available or not tolerated. Treatment in subsequent lines of therapy depends on the first-line therapy or on T790M mutation status. Treatment recommendations for patients whose tumours harbour uncommon EGFR mutations are guided mainly by retrospective and limited prospective evidence. Finally, the evidence for sequencing and combining TKIs with chemotherapy, angiogenesis inhibitors, checkpoint inhibitors, and other new therapeutics is reviewed.

Conclusions This Canadian expert consensus statement and algorithm were driven by significant advances in the treatment of EGFR-mutated NSCLC.

Key Words Non-small-cell lung cancer, advanced; NSCLC; EGFR mutation; acquired resistance; sequencing; mutations, common; mutations, uncommon; algorithms

\section{BACKGROUND}

Few therapeutic areas have experienced as much progress in recent times as EGFR-mutated non-small-cell lung cancer (NSCLC). An estimated $15 \%$ of Canadians with NSCLC tumours have an activating mutation in the EGFR gene in exons 18-21, the region encoding the tyrosine kinase domain ${ }^{1}$. Since the introduction of epidermal growth factor receptor (EGFR) tyrosine kinase inhibitors (TKIs) in 2010 in
Canada, EGFR mutation testing for patients with advanced nonsquamous NSCLC has been the standard of care, with results prompting a specific treatment algorithm for this subset of lung cancer.

More frequent in lifetime never-smokers, the most common EGFR mutations are the exon 19 deletion (exon 19del)

Except for the first and last authors, authors are presented in alphabetic order. 
and the exon 21 codon 858 point mutation (L858R) $)^{1}$. Uncommon mutations, also found in exons 18-21, account for the remaining $8 \%-18 \%$ of all EGFR mutations and might be more prevalent in men and smokers ${ }^{1-3}$. Among the uncommon mutations are sensitizing EGFR mutations-for example, G719X, S768I, and L861Q-and the TKI resistant mutations-including exon 20 insertions and de novo exon 20 T790M mutations.

The EGFR TKIs have activity in tumours harbouring sensitizing EGFR gene mutations, including the first-generation TKIs erlotinib and gefitinib and the second-generation irreversible binding EGFR TKIs afatinib and dacomitinib. Multiple studies ${ }^{4-13}$ and meta-analyse ${ }^{14-16}$ demonstrate the improved efficacy of the first- and second-generation EGFR TKIs compared with chemotherapy in delaying disease progression in first-line treatment. The third-generation EGFR TKI osimertinib was designed for, and found to selectively inhibit, tumours with the acquired T790M resistance mutation, but it also remained active against disease featuring common exon 19del and L858R EGFR mutations, while having less activity against wild-type EGFR. EGFR exon 20 insertions are a class on their own and have been particularly difficult to treat with new therapies under development ${ }^{2,3,17}$.

Guidelines recognize EGFR TKIs as the standard of care for the treatment of advanced EGFR-mutated NSCLC. New developments in systemic therapy include new targeted agents, a better understanding of EGFR TKI resistance mechanisms and how to overcome them, and novel combinations of agents. In this paper, we present an evidence-based treatment algorithm to guide therapeutic choices in the Canadian context.

\section{METHODS}

An invited expert panel of thoracic medical oncologists was tasked with identifying key clinical questions, critically reviewing recent evidence related to the systemic treatment of EGFR-mutated NSCLC, and summarizing evidenceinformed treatment recommendations. The first author drafted the manuscript with the assistance of a medical writer, and all authors provided feedback and revisions. Authors documented their level of agreement to each of the clinical recommendations using a 5-point Likert scale (5, strongly agree; 4 , agree; 3 , neutral; 2 , disagree; 1 , strongly disagree). The final level of agreement was an average of the responses from all authors. All authors approved the final manuscript and algorithm.

\section{CLINICAL QUESTIONS, RECOMMENDATIONS, AND EVIDENCE}

The 6 clinical questions and related recommendations are presented in the subsections that follow, together with a brief summary of the evidence for each point. The final recommendations are reflected in the algorithm shown in Figure 1.

\section{Clinical Question 1}

What is the optimal first-line treatment for patients with advanced NSCLC whose tumours harbour common EGFR mutations?

\section{Recommendation 1a}

Osimertinib is the preferred first-line treatment for patients with advanced NSCLC whose tumours harbour common EGFR mutations.

— Level of consensus: $4.875(7 \times 5,1 \times 4)$

Evidence: The phase III FLAURA trial randomized 556 patients with NSCLC having common EGFR exon 19del and L858R mutations to receive either osimertinib or a first-generation EGFR TKI (gefitinib or erlotinib) ${ }^{17}$. The study demonstrated a significant improvement in overall survival (OS) with osimertinib, the median os being 38.6 months [95\% confidence interval (CI): 34.5 months to 41.8 months] compared with 31.8 months with gefitinib or erlotinib [95\% CI: 26.6 months to 36.0 months; hazard ratio (HR): 0.80 ; 95.05\% CI: 0.64 to $1.00 ; p=0.046]^{18}$. That result is consistent with an earlier publication of the study showing superior, but nonsignificantly improved, os with osimertinib ${ }^{19}$. Although the objective response rates (ORRs) were similar in the two arms, the duration of response was prolonged with osimertinib, at 17.2 months (95\% CI: 13.8 months to 22.0 months) compared with 8.5 months with erlotinib or gefitinib (95\% CI: 7.3 months to 9.8 months) ${ }^{19}$. Grade 3 or greater adverse events were less frequent with osimertinib.

A subgroup analysis in FLAURA showed that, despite experiencing a significant progression-free survival (PFS) benefit, patients of Asian ethnicity did not experience an os benefit ${ }^{17}$. However, that exploratory analysis was not powered to show os differences. Given the strong PFs benefit observed in that subgroup, specific groups are, until further data are available, not excluded from our recommendation of osimertinib as the preferred EGFR TKI.

Providing a third-generation agent in the first-line setting might lead to concerns about restricted options in later lines of therapy; however, up to $30 \%$ of patients with EGFR-mutated NSCLC as seen in the FLAURA trial never received a second-line therapy ${ }^{18}$.

\section{Recommendation $1 b$}

First- and second-generation EGFR TKIs are reasonable first-line options for patients with advanced NSCLC whose tumours harbour common EGFR mutations (exon 19del or L858R) when osimertinib is not available or for patients who had to discontinue osimertinib because of an adverse event. —Level of consensus: 5 (unanimous)

Evidence: The efficacy of the second-generation EGFR TKIs afatinib and dacomitinib, compared with gefitinib, in the first-line setting for patients with exon 19del and L858R EGFR-mutated NSCLC were demonstrated in the LUX-Lung 7 and ARCHER 1050 trials respectively.

The phase IIB randomized LUX-Lung 7 trial demonstrated a statistically significant improvement in PFS with afatinib (HR: $0.73 ; 95 \%$ CI: 0.57 to $0.95 ; p=0.017$ ) and prolonged median time to treatment failure (HR: 0.73 ; $95 \%$ CI: 0.58 to $0.92 ; p=0.0073$ ). However, afatinib did not demonstrate a statistically significant os advantage (27.9 months with afatinib vs. 24.5 months with gefitinib; HR: 0.86; $95 \%$ CI: 0.66 to $1.12 ; p=0.26)^{20,21}$. The trial was insufficiently powered for a proper os evaluation. 
The phase III ARCHER 1050 trial excluded patients with brain metastasis ${ }^{22}$. Although it reported a numeric improvement in os of 34.1 months for dacomitinib (95\% CI: 29.5 months to 37.7 months) compared with 26.8 months for gefitinib (95\% CI: 23.7 months to 32.1 months; HR: 0.760; 95\% CI: 0.582 to 0.993$)$, the trial had a hierarchical stratified statistical design, and given that it did not meet significance in ORR, a $p$ value could not be given to $\mathrm{Os}^{23}$.

The toxicity profiles of second-generation agents vary. Their relative effects are described or discussed in detail elsewhere $^{19,20,24}$, but are generally worse than those for the first-generation EGFR TKIs, resulting in more frequent dose reductions. When selecting between a first- and second-generation EGFR TKI, the toxicity profile should be considered in patient-specific decisions.

An important unresolved question is whether sequencing maximizes survival: Could initial treatment with a second-generation EGFR TKI, followed by osimertinib, compared with upfront osimertinib in patients with acquired T790M mutations ultimately lead to longer survival for the entire group? Unfortunately, randomized data to answer that question are not available. Based on
LUX-Lung 7, patients who received afatinib followed by a third-generation TKI experienced prolonged survival, with os not reached at 4 years ${ }^{25,26}$. The reported rate of T790M mutation after gefitinib, erlotinib, and afatinib ranges from $40 \%$ to $60 \%$ and occurs in up to $73 \%$ of patients with an exon 19del mutation ${ }^{27-29}$. Data for the rate after dacomitinib are insufficient. Ultimately, it is impossible to know who will and will not acquire T790M secondary mutations. Nonetheless, a global debate continues.

\section{Recommendation 1c}

Inhibitors of PD-1/-L1 as single agents or in combination with a platinum doublet do not have a role in the first-line treatment of EGFR-mutated NSCLC, and PD-L1 status should not be used to select first-line treatment for EGFR-mutated NSCLC. — Level of consensus: 5 (unanimous)

Evidence: Recommendation 1c is based on 0 responses seen in a phase II trial of pembrolizumab in PD-L1-positive EGFR-mutated NSCLC in the first-line setting that was closed for futility. A potentially increased risk of autoimmune toxicity (pneumonitis and hepatitis) was also observed in

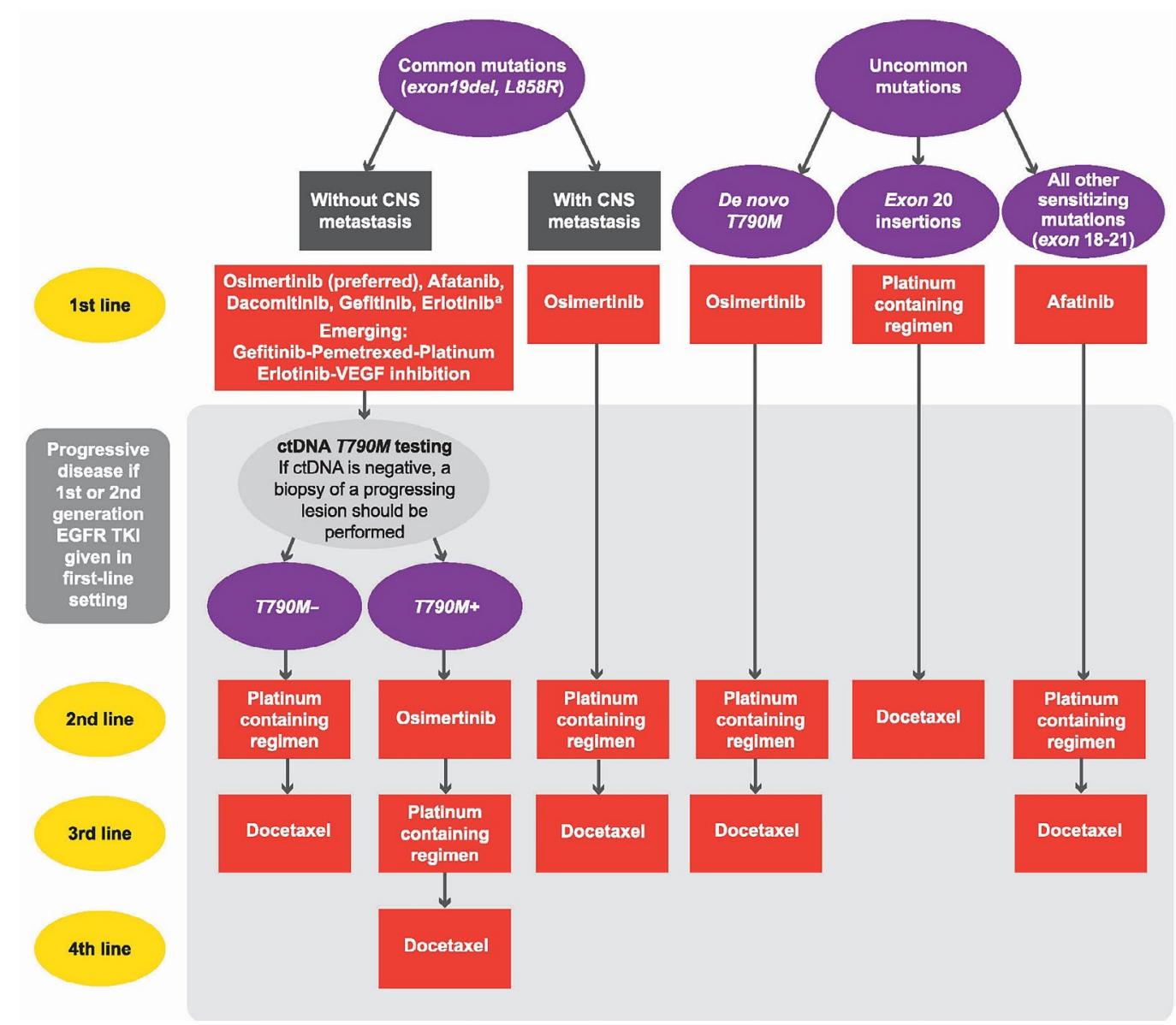

FIGURE 1 Canadian algorithm for the systemic treatment of patients with advanced EGFR-mutation positive non-small-cell lung cancer (NSCLC), from initial diagnosis of advanced disease, with confirmation of the mutation. The figure includes patients with common and uncommon mutations and with and without central nervous system metastasis. The grey-shaded area at the bottom of the algorithm represents progressive disease. Clinical trials, if available, are a first choice in all indications and all lines. ${ }^{a}$ Based on FLAURA, gefitinib and erlotinib are not recommended in the first-line setting unless access to osimertinib is limited or unless they are combined with other agents. 
the study when subsequent EGFR TKIs were administered close to or with an immune checkpoint inhibitor (particularly osimertinib) ${ }^{30}$. The panel thus strongly recommends waiting for EGFR results before exposing patients to PD-1/-L1 inhibitors.

High PD-L1 expression does not influence first-line therapy for patients with EGFR-mutated NSCLC because those patients were excluded from all first-line studies of single-agent pembrolizumab ${ }^{31,32}$ and nivolumab ${ }^{33}$, and of combination pembrolizumab-chemotherapy ${ }^{34}$ and nivolumab-ipilimumab ${ }^{35}$. If treatment is urgently required before EGFR molecular results can be obtained, the group suggests starting platinum doublet chemotherapy without a PD-1/-L1 inhibitor.

\section{Clinical Question 2}

What is the preferred first-line systemic treatment for patients with central nervous system (CNS) metastasis?

\section{Recommendation 2a}

Osimertinib is the preferred EGFR TKI for patients with brain metastasis or leptomeningeal disease (LMD).

- Level of consensus: 5 (unanimous)

Evidence: Almost half the patients with metastatic EGFR-mutated NSCLC develop brain metastasis during the course of their disease ${ }^{36,37}$, negatively affecting prognosis and quality of life. Osimertinib is the TKI with the most compelling CNS data ${ }^{38-40}$. The FLAURA trial permitted patients with stable or neurologically asymptomatic CNS metastasis to enrol. However, the protocol contained no directions about how to follow patients without brain metastasis at baseline. Thus, conclusions can be based only on the group with baseline brain metastasis.

After the trial was initiated, an independent review committee was established to evaluate the measurable and non-measurable CNS responses in 61 patients $(22 \%)$ treated with 80 mg osimertinib and in 67 patients $(24 \%)$ treated with either gefitinib or erlotinib (gefitinib/erlotinib) ${ }^{38}$. The CNS PFS assessed by central blinded independent neuroradiologic review was not reached at median in the osimertinib arm [95\% CI: 16.5 months to not calculable (NC)]; in the gefitinib/erlotinib arm, the CNS PFS was 13.9 months (95\% CI: 8.3 months to NC). A statistically significant and clinically relevant improvement in CNS PFS was observed for osimertinib compared with gefitinib/erlotinib (HR: 0.48; $95 \%$ CI: 0.26 to $0.86 ; p=0.014)^{38}$. In patients with 1 or more measurable CNS lesions, the CNS ORR was $91 \%$ for osimertinib and $68 \%$ for gefitinib/erlotinib. The median CNS PFS with osimertinib was not reached (95\% CI: 16.5 months to $\mathrm{NC}$ ); it was 13.9 months for gefitinib/erlotinib (95\% CI: 8.3 to NC; HR: 0.48$)$.

In the AURA3 study, the CNS ORR was measured as part of a preplanned subgroup analysis ${ }^{39}$. Of 116 patients with measurable or unmeasurable brain metastasis, median CNS PFS was longer for patients receiving $80 \mathrm{mg}$ osimertinib than for those receiving chemotherapy, at 11.7 months compared with 5.6 months respectively, by blinded independent review committee (HR: $0.32 ; 95 \%$ CI: 0.15 to $0.69 ; p=0.004)^{39}$. Of 46 patients with 1 or more measurable cNS metastases, 30 patients had been treated with osimertinib, with 21 achieving a response (ORR: $70 \%$; $95 \%$ CI: $51 \%$ to $85 \%$ ), and 16 had been treated with chemotherapy, with 5 achieving a response (ORR: $31 \%$; $95 \%$ CI: $11 \%$ to $59 \%)^{39}$.

Osimertinib has also been studied in patients with LMD. In the phase I BLOOM study, 21 patients who had LMD and stable extracranial disease, and who had received an EGFR TKI, were treated with $160 \mathrm{mg}$ osimertinib daily ${ }^{41}$. Radiologic improvement was confirmed in 7 , and 9 had stable disease. The trial did not compare the $80 \mathrm{mg}$ and $160 \mathrm{mg}$ doses. A more recent evaluation of 22 patients with LMD in the AURA program of $80 \mathrm{mg}$ osimertinib daily, reported an ORR of $55 \%$ (95\% CI: $32 \%$ to $76 \%$ ). The median LMD PFS and os were 11.1 months (95\% CI: 4.6 months to NC) and 18.8 months (95\% CI: 6.3 months to NC) respectively ${ }^{42}$.

Thus, the group recommends standard-dose osimertinib $80 \mathrm{mg}$ for patients with CNS LMD metastasis until further studies are conducted to evaluate the additional benefit of the increased dose of $160 \mathrm{mg}$. There are no data to guide the treatment of patients with CNS progression after osimertinib treatment, and there is no evidence that dose escalation would improve patient outcomes.

All TKIs have demonstrated responses in CNS metastases in retrospective case series ${ }^{43}$. Afatinib delayed the onset or progression of brain metastasis in a pooled analysis of the LuX-Lung 3, 6, and 7 studies ${ }^{41}$ and in a real-world population of patients with advanced NSCLC ${ }^{44}$. The ARCHER 1050 study of dacomitinib excluded patients with CNS metastasis at baseline and did not mandate imaging of the brain, thus providing no evidence of whether dacomitinib prevents the development of brain metastasis ${ }^{23}$. However, the primary site of progression was noted for patients who progressed while on trial, and a brain site was recorded in only 1 of 227 patients in the dacomitinib arm and in 11 of 225 patients in the gefitinib $\mathrm{arm}^{23}$.

\section{Clinical Question 3}

What evidence do we have for treating patients whose tumours harbour uncommon EGFR mutations?

Uncommon EGFR mutations are heterogeneous, with various insertions or base substitutions observed in exons 18-21, and they can often be compound or complex, with 2 different mutations being present in the same tumour $^{2,3}$. Patients with uncommon EGFR mutations are often excluded from clinical trials and primary analyses, and so much of what is known about their response to EGFR TKIs comes from post hoc ${ }^{17}$ or retrospective analyses of trial data $^{2}$ or from clinical practice ${ }^{3}$. Uncommon mutations have historically been categorized into 3 subgroups based on in vitro and in vivo response to EGFR TKIs:

\section{EGFR sensitizing mutations (G719X, S768I, and L861Q) Exon 20 insertions \\ De novo T790M mutations.}

\section{Recommendation $3 a$}

Afatinib is the preferred first-line treatment for patients with uncommon EGFR TKI sensitizing mutations (point mutations or duplications in exons 18-21, excluding T790M and exon 20 insertions).

— Level of consensus: $4.125(1 \times 5,7 \times 4)$ 
Evidence: The highest level of prospective data comes from studies of afatinib. Most evidence focuses on patients with the exon 18 G719X, exon 20 S768I, and exon 21 L861Q somatic mutations. For patients with tumours carrying G719X, S768I, or L861Q mutations treated with afatinib, median os durations were, respectively, 26.9 months $(95 \%$ CI: months 16.4 to not evaluable), not evaluable (95\% CI: 3.4 months to not evaluable), and 17.1 months (95\% CI: 15.3 months to 21.6 months). For the LuX-Lung 2, 3, and 6 trials, a pooled analysis ${ }^{17}$ showed responses similar to those in patients having standard EGFR mutations ${ }^{3}$.

Studies of osimertinib in patients with uncommon mutations are ongoing. Patients with uncommon mutations were excluded from the FLAURA trial. Interim results of a phase II single arm study of patients with treatment-naïve $(61 \%)$ and pretreated disease demonstrated that osimertinib was active: the ORR in patients with L861Q was $77.8 \%$; with G719X, 52.8\%; and with S768I, 37.5\%; the median PFS was only 9.5 months (range: $1.0-20.1$ months) ${ }^{45}$, however. The ASTRIS real-world treatment study measured outcomes in 53 patients with an acquired T790M mutation in the setting of uncommon mutations; those patients were treated with 1 or more lines of EGFR TKI followed by osimertinib, resulting in a median PFS of 8.1 months compared with the 11.1 months seen in the full dataset $(n=3015)^{46,47}$. Studies in patients with uncommon EGFR mutations who were naïve to EGFR TKIs are continuing (see NCT03434418 at https:// ClinicalTrials.gov/).

\section{Recommendation $3 b$}

Osimertinib is the preferred first-line treatment for patients with de novo T790M mutation.

- Level of consensus: 5 (unanimous)

Evidence: De novo T790M mutations occurring without prior EGFR TKI treatment are rare and result in a poor response to first- and second-generation EGFR TKIs ${ }^{3}$. Patients with germline T790M mutations might have an inherited propensity for lung cancer ${ }^{48}$. All patients with a de novo T790M mutation should also be referred to a hereditary cancer program, because standard tumour-based assays do not differentiate between a de novo somatic mutation and a germline mutation. Treatment evidence is lacking, and so patients with a de novo T790M mutation are usually empirically treated similarly to those with an acquired T790M mutation ${ }^{1}$.

\section{Recommendation 3c}

Patients with an exon 20 insertion (excluding T790M) should be treated with a platinum doublet or on a clinical trial. - Level of consensus: $4(4 \times 5,1 \times 4,2 \times 3,1 \times 2)$

Evidence: Patients with an exon 20 insertion are resistant to first- and second-generation EGFR TKIs ${ }^{2,3,17}$. Response to osimertinib has been reported in case studies, but the largest series to date suggests, at most, a $7.5 \%$ response rate $^{49}$. The preferred treatment is a platinum doublet. As an alternative, numerous clinical trials with promising targeted therapeutics specifically designed to inhibit such mutations are in development ${ }^{50}$, including poziotinib ${ }^{51,52}$, TAK- $788^{53}$, tarloxotinib ${ }^{54}$, and the JNJ-372 bi-specific antibody ${ }^{55}$. Whether affected patients should receive atezolizumab-bevacizumab-carboplatin-paclitaxel (АВCP) remains controversial (lack of consensus to recommend for or against); the regimen is under active investigation (see NCT03991403 at https://ClinicalTrials.gov/).

\section{Clinical Question 4}

What is the preferred second-line treatment for patients who received a first- or second-generation TKI in the firstline setting?

The development of acquired resistance is a key challenge in treating patients with EGFR-mutated NSCLC. The most common resistance mechanism in patients treated with first- and second-generation EGFR TKIs is an acquired EGFR T790M (exon 20) mutation that changes a conserved threonine residue in the ATP binding pocket to methionine and that occurs in $50 \%-60 \%$ of cases of acquired resistance $^{56-58}$. The mutation results in a conformational change of the kinase binding pocket and prevents the activity of the inhibitors. The individuals who will develop a T790M mutation after progression on first- and second-generation EGFR TKIs cannot be predicted; thus, all patients treated with a first- or second-generation TKI for common or uncommon mutations have to undergo T790M molecular testing on progression. Testing guidelines are beyond the scope of the present document and are published elsewhere ${ }^{59}$.

\section{Recommendation $4 a$}

Patients who received a first- or second-generation EGFR TKI in the first-line setting and who are T790M-positive should be treated with osimertinib or on a clinical trial. - Level of consensus: 5 (unanimous)

Evidence: Several trials demonstrated the benefit of a third-generation TKI after progression on a first- or secondgeneration TKI. The phase III AURA3 trial randomized 419 patients with an acquired T790M mutation after progression on a first- or second-generation EGFR TKI to osimertinib versus chemotherapy (pemetrexed-carboplatin or pemetrexed-cisplatin) ${ }^{60}$. The primary endpoint of investigator-assessed PFS was significantly longer for osimertinib compared with chemotherapy (10.1 months vs. 4.4 months; HR: $0.30 ; 95 \%$ CI: 0.23 to $0.41 ; p<0.001)$. The ORR was $71 \%$ for osimertinib (95\% cI: $65 \%$ to $76 \%$ ) compared with $31 \%$ for chemotherapy ( $95 \%$ cI: $24 \%$ to $40 \%$; odds ratio for ORR: 5.39 ; 95\% CI: 3.47 to $8.48 ; p<0.001$ ). Adverse events of grade 3 or greater were less frequent with osimertinib (23\%) than with chemotherapy (47\%). Median os was 26.8 months in the osimertinib arm (95\% CI: 23.5 months to 31.5 months) compared with 22.5 months in the chemotherapy arm (95\% CI: 20.2 months to 28.8 months; HR: 0.87; 95\% CI: 0.67 to $1.12 ; p=0.28)^{61}$.

The statistically and clinically significant PFs benefit associated with osimertinib in a comparison with platinum-pemetrexed did not result in a statistically significant improvement in os, possibly as a result of the high crossover rate (patients from the platinum-pemetrexed arm crossing to osimertinib).

Consistent results were observed in a post hoc analysis of the LUX-Lung 7 trial, which examined os in patients 
treated with at least 1 line of post-progression therapy after discontinuing a first- or second-generation $\mathrm{TKI}^{21}$. In patients who received a third-generation TKI at some time as subsequent treatment, median os was 48.3 months for patients initially treated with gefitinib and is still not reached for those initially treated with afatinib ${ }^{26}$.

The benefit of subsequent second- and third-generation TKIs has also been demonstrated in the GioTag real-world study $^{62}$. That retrospective chart review examined 204 patients who had received first-line afatinib followed by second-line osimertinib. The time to failure with firstline afatinib was 11.9 months (90\% CI: 10.9 months to 12.2 months), and with second-line osimertinib, it was 14.3 months ( $90 \%$ CI: 12.8 months to 15.9 months). The combined time to treatment failure was 27.6 months $(90 \%$ CI: 25.9 months to 31.3 months). At data cut-off, $48 \%$ of the patients were still on treatment, with a 2 -year os of $79 \%$ and a 30 -month survival rate of $69 \%^{62}$.

\section{Recommendation $4 b$}

In patients who are progressing after a first-line first- or second-generation inhibitor and who are T790M-negative, the preferred second-line treatment is a platinum doublet or a clinical trial. Whether patients should receive $A B C P$ or other chemotherapy combinations [anti-PD-1, anti-vascular endothelial growth factor (VEGF)] remains controversial (no consensus to recommend for or against); the regimen is under active investigation.

- Level of consensus: $4.125(2 \times 5,5 \times 4,1 \times 3)$

Evidence: Clinical trials are needed to develop more compelling recommendations for this patient population. Patients who do not acquire a T790M mutation at progression might develop alternative EGFR-dependent and -independent resistance mechanisms, including phenotypic transformation, HER 2 and $M E T$ amplification or activation, and activation of Raf, Ras, or VEGFR pathways ${ }^{59,63}$. If available, comprehensive gene testing should be considered to look for alternative gene resistance mechanisms and the potential for clinical trial enrolment.

In the absence of trials, these patients should receive platinum-doublet chemotherapy as the standard or be enrolled on trials of chemotherapy combinations (for example, 4-drug or 3-drug regimens) or targeted therapies. The IMpower150 trial tested the combination of bevacizumab, an antibody against VEGF, or atezolizumab, a PD-L1 antibody, with a carboplatin-paclitaxel chemotherapy backbone (BCP, ACP), or the chemotherapy backbone with both antibodies (ABCP) ${ }^{64}$. In a post hoc analysis, a trend toward longer os was observed in patients with EGFR-mutant lung cancer when treated with the quadruplet (HR for ABCP compared with BCP: $0.61 ; 95 \%$ CI: 0.29 to 1.28 ), although the Kaplan-Meier curves favoured BCР in the first 4 months ${ }^{65}$. When only the 57 patients with EGFR sensitizing mutations, previously treated with a TKI, were analyzed, the survival HR was 0.31 favouring ABCP over BCP (95\% CI: 0.11 to 0.83 ). Those data are compelling; however, based on existing evidence, a consensus could not be reached to recommend for or against $\mathrm{ABCP}$. A larger randomized study testing that question in patients with $E G F R$ - or $A L K$-driven lung cancer is ongoing.

\section{Clinical Question 5}

What is the preferred treatment after osimertinib?

In the first-line FLAURA trial, none of the patients treated with first-line osimertinib acquired the T790M mutation ${ }^{66}$.

Patients treated with osimertinib develop resistance through several different mechanisms, including MET amplification $^{66}$, acquisition of an exon 20 EGFR C797S point mutation $^{66}$, or loss of the T790M mutation (in treated patients with already-acquired resistance), which does not re-sensitize patients to a first- or second-generation EGFR TKI $^{67}$. The field is complex, and research into targeting resistant mutations is ongoing ${ }^{68}$.

\section{Recommendation 5 a}

For patients treated with osimertinib in the first-line setting or in the second line after a first- or second-generation EGFR TKI, the preferred subsequent systemic treatment is a platinum doublet or a clinical trial.

— Level of consensus: $4.375(5 \times 5,1 \times 4,2 \times 3)$

A platinum doublet or clinical trial is the preferred option. The panel could not reach consensus for or against platinum plus an anti-PD-1 antibody or ABCP in this setting. Other options to consider in patients with a poor performance status include single-agent chemotherapy with pemetrexed, gemcitabine, or vinorelbine. For those with oligoprogressive disease, participation in trials of local therapy such as radiation-for example, STOP (see NCT02756793 at https:// ClinicalTrials.gov/)—should be considered.

Evidence: Multiple clinical trials are exploring strategies to overcome both EGFR-dependent and -independent resistance mechanisms. Those trials include next-generation TKIs $^{63,69-72}$, TKIs designed to target multiple mutations ${ }^{73}$, dual pathway inhibition ${ }^{72}$, combinations of EGFR TKIs with other agents ${ }^{74-81}$, and novel agents ${ }^{55,82}$.

\section{Upcoming Strategies}

The upcoming strategies discussed here had not yet been approved by Health Canada at the time of writing.

\section{Recommendation 6a}

The combination of gefitinib plus chemotherapy could be considered in the first-line setting for patients not eligible for osimertinib or a clinical trial.

— Level of consensus: $4(1 \times 5,6 \times 4,1 \times 3)$

Evidence: Two randomized phase III trials demonstrated improved clinical outcomes for gefitinib plus chemotherapy compared with gefitinib monotherapy in treatment-naïve EGFR-mutated NSCLC.

The Japanese NEJ009 phase III study randomized 344 patients with common EGFR mutations to gefitinib or to gefitinib-carboplatin-pemetrexed, followed by maintenance pemetrexed ${ }^{83,84}$. Although PFs was significantly better and median os was numerically higher in the TKIchemotherapy arm than in the gefitinib-alone arm, PFs2 was similar in the two arms and did not meet the endpoint necessary to formally analyze os because of a hierarchical statistical testing design. Most of the patients in the trial 
crossed over, and $22 \%-30 \%$ had brain metastasis, a group that normally experiences poorer outcomes.

A study from Mumbai, India, randomized 350 patients with sensitizing EGFR mutations (including uncommon ones) to gefitinib or to gefitinib-pemetrexed-carboplatin followed by pemetrexed maintenance ${ }^{85}$. The study included patients with treated brain metastases (18\% of patients) and those with an Eastern Cooperative Oncology Group performance status of 2, who were excluded from the FLAURA trial. The addition of chemotherapy to the EGFR TKI was associated with significantly prolonged $P F S$ and $0 S^{85}$. At disease progression, the crossover rate from the gefitinib arm to chemotherapy was $32.4 \%$. With adoption of the combination approach, higher toxicities were described.

\section{Recommendation $6 b$}

No consensus could be reached for or against a consideration of combining erlotinib with VEGF inhibition in the first-line setting.

- Level of consensus: $3(2 \times 4,4 \times 3,2 \times 2)$

Evidence: Combinations of erlotinib with bevacizumab (anti-VEGF antibody) or with ramucirumab (anti-VEGF receptor antibody) are promising and have been associated with PFS improvements in first-line trials, including JO25567 (phase II) ${ }^{86}$, NEJ026 (phase III) ${ }^{87}$, and RELAY (randomized double-blind phase III ${ }^{88}$. Although os differences were insignificant in the JO25567 trial $^{89}$, final os data from the NEJ026 and RELAY trials are awaited before a role as a firstline treatment option can be defined for erlotinib-VEGF inhibitors. The data will also have to be assessed in context, because the comparator arm is erlotinib, which has an os inferior to that with osimertinib, the new standard of care. Additional trials in this area are ongoing.

\section{DISCUSSION AND CONCLUSIONS}

Patients with EGFR-mutated NSCLC currently have many systemic treatment options available, and we encourage enrolment onto clinical trials when possible. Upcoming clinical trial results and the emergence of new agents and combinations will likely change the algorithm presented here. We look forward to potentially better survival options for our patients.

\section{ACKNOWLEDGMENTS}

This article was supported by grants from AstraZeneca Canada, Boehringer Ingelheim Canada, Hoffman-La Roche Canada, Merck Canada, Pfizer Canada, and Takeda Canada. Those entities did not influence the content of the article, nor did they review the article before publication. The authors acknowledge the assistance of medical writer Dr. Chrystal Palaty, Metaphase Health Research Consulting Inc., who was present from conception to the birth of this manuscript.

\section{CONFLICT OF INTEREST DISCLOSURES}

We have read and understood Current Oncology's policy on disclosing conflicts of interest, and we declare the following interests: $\mathrm{BM}$ has received honoraria for advisory board participation from Bayer, Pfizer, AstraZeneca, Boehringer Ingelheim, and Roche. SB has received honoraria for advisory board participation from AstraZeneca, Bayer, Boehringer Ingelheim, Bristol-Myers Squibb, GlaxoSmithKline, Lilly, Merck, Novartis, Pfizer, Roche, and Takeda. NB has received honoraria for advisory board participation from AstraZeneca, Bayer, Boehringer Ingelheim, Bristol-Myers Squibb, GlaxoSmithKline, Lilly, Merck, Novartis, Pfizer, Roche, and Takeda, and has received research funding from AstraZeneca. QC has received honoraria for advisory board participation from AbbVie, AstraZeneca, Bayer, Boehringer Ingelheim, Bristol-Myers Squibb, Eisai, Eli Lilly, Merck, Novartis, Pfizer, Roche, and Takeda. RJ has received honoraria for advisory board participation from AstraZeneca, Bristol-Myers Squibb, Fusion, Merck, Novartis, Pfizer, Roche, and Takeda, and research funding from AstraZeneca, Bristol-Myers Squibb, and Merck. NBL is a beneficiary of institutional research funding from Guardant, Array, Novartis, Merck, and Roche; has received honoraria or travel expenses for independent continuing medical education from Roche, AstraZeneca, Merck Sharp and Dohme, and Bristol-Myers Squibb; and has acted as an advisor (compensated) for Xcovery and as a consultant for the Canadian Agency for Drugs and Technologies in Health. GL has received honoraria for advisory board participation from AstraZeneca, Roche, Pfizer, Takeda, Novartis, Merck, Bristol-Myers Squibb, Boehringer Ingelheim, and Bayer, and for providing education consulting services to AstraZeneca, Roche, EMD Serono, and Takeda. GL has also received research funding from AstraZeneca, Roche, and Takeda. PC has received honoraria for advisory board participation from AstraZeneca, Boehringer Ingelheim, Roche, Pfizer, Takeda, Merck, and BristolMyers Squibb.

\section{AUTHOR AFFILIATIONS}

*BC Cancer-Vancouver Centre, Vancouver, BC; ${ }^{\dagger}$ CancerCare Manitoba, University of Manitoba, Winnipeg, $\mathrm{MB} ;{ }^{\ddagger}$ Centre hospitalier del'Université de Montréal, Montreal, QC; ${ }^{\circledR}$ Cross Cancer Institute, Edmonton, AB; "McMaster University, Juravinski Cancer Centre, Hamilton, ON; "Princess Margaret Cancer Centre, University of Toronto, Toronto, ON; **William Osler Health System, University of Toronto, Brampton, ON.

\section{REFERENCES}

1. Graham RP, Treece AL, Lindeman NI, et al. Worldwide frequency of commonly detected EGFR mutations. Arch Pathol Lab Med 2018;142:163-7.

2. O'Kane GM, Bradbury PA, Feld R, et al. Uncommon EGFR mutations in advanced non-small cell lung cancer. Lung Cancer 2017;109:137-44.

3. Tu HY, Ke EE, Yang JJ, et al. A comprehensive review of uncommon EGFR mutations in patients with non-small cell lung cancer. Lung Cancer 2017;114:96-102.

4. Rosell R, Carcereny E, Gervais R, et al. on behalf of the Spanish Lung Cancer Group in collaboration with the Groupe français de pneumo-cancérologie and the Associazione Italiana Oncologia Toracica. Erlotinib versus standard chemotherapy as first-line treatment for European patients with advanced EGFR mutation-positive non-small-cell lung cancer (EURTAC): a multicentre, open-label, randomised phase 3 trial. Lancet Oncol 2012;13:239-46.

5. Zhou C, Wu YL, Chen G, et al. Erlotinib versus chemotherapy as first-line treatment for patients with advanced EGFR mutation-positive non-small-cell lung cancer (OPTIMAL, CTONG-0802): a multicentre, open-label, randomised, phase 3 study. Lancet Oncol 2011;12:735-42.

6. Maemondo M, Inoue A, Kobayashi K, et al. Gefitinib or chemotherapy for non-small-cell lung cancer with mutated $E G F R$. NEngl J Med 2010;362:2380-8.

7. Mitsudomi T, Morita S, Yatabe Y, et al. Gefitinib versus cisplatin plus docetaxel in patients with non-small-cell lung cancer harbouring mutations of the epidermal growth factor receptor (WJTOG3405): an open label, randomised phase 3 trial. Lancet Oncol 2010;11:121-8. 
8. MokTS, WuYL, ThongprasertS, etal. Gefitinib or carboplatinpaclitaxel in pulmonary adenocarcinoma. NEnglJMed 2009; 361:947-57.

9. Fukuoka M, Wu YL, Thongprasert S, etal. Biomarker analyses and final overall survival results from a phase III, randomized, open-label, first-line study of gefitinib versus carboplatin/ paclitaxel in clinically selected patients with advanced nonsmall-cell lung cancer in Asia (IPASS). J Clin Oncol 2011;29: 2866-74.

10. Sequist LV, Yang JC, Yamamoto N, et al. Phase III study of afatinib or cisplatin plus pemetrexed in patients with metastatic lung adenocarcinoma with EGFR mutations. J Clin Oncol 2013;31:3327-34.

11. Yang JC, Wu YL, Schuler M, et al. Afatinib versus cisplatin-based chemotherapy for EGFR mutation-positive lung adenocarcinoma (LUX-Lung 3 and LUX-Lung 6): analysis of overall survival data from two randomised, phase 3 trials. Lancet Oncol 2015;16:141-51.

12. Wu YL, Zhou C, Hu CP, et al. Afatinib versus cisplatin plus gemcitabine for first-line treatment of Asian patients with advanced non-small-cell lung cancer harbouring EGFR mutations (LuX-Lung 6): an open-label, randomised phase 3 trial. Lancet Oncol 2014;15:213-22.

13. Wu YL, Zhou C, Liam CK, et al. First-line erlotinib versus gemcitabine/cisplatin in patients with advanced EGFR mutation-positive non-small-cell lung cancer: analyses from the phase III, randomized, open-label, ENSURE study. Ann Oncol 2015;26:1883-9.

14. Lee CK, Brown C, Gralla RJ, et al. Impact of EGFR inhibitor in non-small cell lung cancer on progression-free and overall survival: a meta-analysis. J Natl Cancer Inst 2013;105:595-605.

15. Gao G, Ren S, Li A, et al. Epidermal growth factor receptortyrosine kinase inhibitor therapy is effective as first-line treatment of advanced non-small-cell lung cancer with mutated EGFR: a meta-analysis from six phase III randomized controlled trials. Int J Cancer 2012;131:E822-9.

16. Bria E, Milella M, Cuppone F, et al. Outcome of advanced NSCLC patients harboring sensitizing EGFR mutations randomized to EGFR tyrosine kinase inhibitors or chemotherapy as first-line treatment: a meta-analysis. Ann Oncol 2011;22:2277-85.

17. Yang JC, Sequist LV, Geater SL, et al. Clinical activity of afatinib in patients with advanced non-small-cell lung cancer harbouring uncommon EGFR mutations: a combined posthoc analysis of LUX-Lung 2, LUX-Lung 3, and LUX-Lung 6. Lancet Oncol 2015;16:830-8.

18. Ramalingam SS, Vansteenkiste J, Planchard D, et al. Overall survival with osimertinib in untreated, EGFR-mutated advanced NSCLC. N Engl J Med 2020;382:41-50.

19. Soria JC, Ohe Y, Vansteenkiste J, et al. on behalf of the FLAURA investigators. Osimertinib in untreated EGFRmutated advanced non-small-cell lung cancer. N Engl J Med 2018;378:113-25.

20. Park K, Tan EH, O'Byrne K, et al. Afatinib versus gefitinib as first-line treatment of patients with EGFR mutation-positive non-small-cell lung cancer (LUX-Lung 7): a phase 2B, openlabel, randomised controlled trial. Lancet Oncol 2016;17:577-89.

21. Paz-Ares L, Tan EH, O'Byrne K, et al. Afatinib versus gefitinib in patients with EGFR mutation-positive advanced non-small-cell lung cancer: overall survival data from the phase IIB LUX-Lung 7 trial. Ann Oncol 2017;28:270-7.

22. Wu YL, Cheng Y, Zhou X, et al. Dacomitinib versus gefitinib as first-line treatment for patients with EGFR-mutation-positive non-small-cell lung cancer (ARCHER 1050): a randomised, open-label, phase 3 trial. Lancet Oncol 2017;18:1454-66.

23. Mok TS, Cheng Y, Zhou X, et al. Improvement in overall survival in a randomized study that compared dacomitinib with gefitinib in patients with advanced non-small-cell lung cancer and EGFR-activating mutations. J Clin Oncol 2018; 36:2244-50.

24. Mok T, Cheng Y, Zhou X, et al. Dacomitinib versus gefitinib for the first-line treatment of advanced EGFR mutation positive non-small cell lung cancer (ARCHER 1050): a randomized, open-label phase III trial [abstract LBA9007]. J Clin Oncol 2017;35:. [Available online at: https://ascopubs.org/doi/ abs/10.1200/JCO.2017.35.18_suppl.LBA9007; cited 17 April 2020]

25. Hochmair MJ, Morabito A, Hao D, et al. Sequential afatinib and osimertinib in patients with EGFR mutation-positive non-small-cell lung cancer: updated analysis of the observational GioTag study. Future Oncol 2019;15:2905-14.

26. Corral J, Zhang L, Park K, et al. Afatinib (A) vs gefitinib (G) in patients with EGFR mutation-positive (EGFRm+) NSCLC: updated os data from the phase IIB trial LUX-Lung 7 (LL7) [abstract 93PD]. Ann Oncol 2017;28(suppl 2):.

27. Jenkins S, Yang JC, Jänne PA, et al. EGFR mutation analysis for prospective patient selection in two phase II registration studies of osimertinib. J Thorac Oncol 2017;12:1247-56.

28. Reungwetwattana T, Gray JE, Markovets A, etal. Longitudinal circulating tumour DNA (ctDNA) monitoring for early detection of disease progression and resistance in advanced NSCLC in FLAURA. Ann Oncol 2019;30 (suppl 9):ix183-202.

29. Hochmair MJ, Buder A, Schwab S, et al. Liquid-biopsy-based identification of EGFRT790M mutation-mediated resistance to afatinib treatment in patients with advanced EGFR mutationpositive NSCLC, and subsequent response to osimertinib. Target Oncol 2019;14:75-83.

30. Ahn MJ, Yang J, Yu H, et al. Osimertinib combined with durvalumab in EGFR-mutant non-small cell lung cancer: results from the TATTON phase IB trial [abstract 136O].J Thorac Oncol 2016;11:S115.

31. Reck M, Rodríguez-Abreu D, Robinson AG, et al. on behalf of the KEYNOTE-024 investigators. Pembrolizumab versus chemotherapy for PD-L1-positive non-small-cell lung cancer. N Engl J Med 2016;375:1823-33.

32. Lopes G, Wu YL, Kudaba I, et al. Pembrolizumab (pembro) versus platinum-based chemotherapy (chemo) as first-line therapy for advanced/metastatic NSCLC with a PD-L1 tumor proportion score (TPS) $\geq 1 \%$ : open-label, phase 3 KEYNOTE-042 study [abstract LBA4]. JClin Oncol 2018;36:. [Available online at: https://ascopubs.org/doi/10.1200/JCO.2018.36.18_suppl. LBA4; cited 17 April 2020]

33. Carbone DP, Reck M, Paz-Ares L, et al. on behalf of the CheckMate 026 investigators. First-line nivolumab in stage IV or recurrent non-small-cell lung cancer. N Engl J Med 2017; 376:2415-26.

34. Gandhi L, Rodríguez-Abreu D, Gadgeel S, et al. on behalf of the KEYNOTE-189 investigators. Pembrolizumab plus chemotherapy in metastatic non-small-cell lung cancer. $N$ Engl $J$ Med 2018;378:2078-92.

35. Hellmann MD, Ciuleanu TE, Pluzanski A, et al. Nivolumab plus ipilimumab in lung cancer with a high tumor mutational burden. N Engl J Med 2018;378:2093-104.

36. Han G, Bi J, Tan W, et al. A retrospective analysis in patients with EGFR-mutant lung adenocarcinoma: is EGFR mutation associated with a higher incidence of brain metastasis? Oncotarget 2016;7:56998-7010.

37. Rangachari D, Yamaguchi N, VanderLaan PA, et al. Brain metastases in patients with $E G F R$-mutated or $A L K$-rearranged non-small-cell lung cancers. Lung Cancer 2015;88:108-11.

38. Reungwetwattana T, Nakagawa K, Cho BC, et al. CNs response to osimertinib versus standard epidermal growth factor receptor tyrosine kinase inhibitors in patients with untreated EGFR-mutated advanced non-small-cell lung cancer. J Clin Oncol 2018;36:3290-7. 
39. Wu YL, Ahn MJ, Garassino MC, et al. CNS efficacy of osimertinib in patients with T790M-positive advanced non-small-cell lung cancer: data from a randomized phase III trial (AURA3). J Clin Oncol 2018;36:2702-9.

40. Ahn MJ, Chiu CH, Cheng Y, et al. Osimertinib for patients (pts) with leptomeningeal metastases (LM) associated with EGFRm advanced NSCLC: the AURA LM study [abstract 1050]. Ann Oncol 2019;30 (suppl 2):ii48.

41. Yang JCH, Wu YL, Hirsh V, et al. Competing central nervous system or systemic progression analysis for patients with EGFR mutation-positive NSCLC receiving afatinib in LUXLung 3, 6, and 7 [abstract 143PD]. JThorac Oncol2018;13(suppl 4):S84-5. [Available online at: https://www.inoncology.com/ sites/default/files/poster/elcc_2018_cns_activity_poster. pdf; cited 30 April 2020]

42. Ahn MJ, Chiu CH, Cheng Y, et al. Osimertinib for patients with leptomeningeal metastases associated with EGFR T790M-positive advanced NSCLC: the AURA leptomeningeal metastases analysis. J Thorac Oncol 2020;15:637-48.

43. Heon S, Yeap BY, Lindeman NI, et al. The impact of initial gefitinib or erlotinib versus chemotherapy on central nervous system progression in advanced non-small cell lung cancer with EGFR mutations. Clin Cancer Res 2012;18:4406-14.

44. Kim Y, Sun J, Park K, et al. First-line afatinib for non-small cell lung cancer in real world practice [abstract P3.01-023]. 2017;12(suppl 2):S2209.

45. Ahn MJ, Cho JH, Sun JM, et al. An open-label, multicenter, phase II single arm trial of osimertinib in non-small cell lung cancer patients with uncommon EGFR mutation (KCSG-LU15-09) [abstract 9050]. J Clin Oncol 2018;36:. [Available online at: https://ascopubs.org/doi/abs/10.1200/ JCO.2018.36.15_suppl.9050; cited 18 April 2020]

46. Marinis F, Wu YL, de Castro GJr, et al. ASTRIS: a global real-world study of osimertinib in $>3000$ patients with $E G F R$ T790M positive non-small-cell lung cancer. Future Oncol 2019;15:3003-14.

47. Cheema P, Passaro A, Martin C, et al. Osimertinib in EGFR T790M advanced NSCLC: analysis of uncommon/complex EGFR mutations in a real-world study (ASTRIS) [P2.14-59]. J Thorac Oncol 2019;14(suppl):S854.

48. Gazdar A, Robinson L, Oliver D, et al. Hereditary lung cancer syndrome targets never smokers with germline EGFR gene T790M mutations. J Thorac Oncol 2014;9:456-63.

49. Fang W, Huang Y, Hong S, et al. EGFR exon 20 insertion mutations and response to osimertinib in non-small-cell lung cancer. BMC Cancer 2019;19:595.

50. Vyse S, Huang PH. Targeting EGFR exon 20 insertion mutations in non-small cell lung cancer. Signal Transduct Target Ther 2019;4:5.

51. Heymach J, Negräo M, Robichaux J, et al. A phase II trial of poziotinib in EGFR and HER2 exon 20 mutant non-small cell lung cancer (NSCLC) [abstract OA02.06]. J Thorac Oncol 2018;13(suppl 10):S323-4.

52. Yang Z, Tchekmedyian N, Chu DT, et al. A phase 2 study of poziotinib in patients with EGFR or HER2 exon 20 mutationpositive non-small cell lung cancer [abstract TPS9106]. J Clin Oncol 2018;36:. [Available online at: https://ascopubs. org/doi/abs/10.1200/JCO.2018.36.15_suppl.TPS9106; cited 18 April 2020]

53. Janne PA, Neal JW, Camidge DR, et al. Antitumour activity of TAK-788 in NSCLC with EGFR exon 20 insertions [abstract 9007]. J Clin Oncol 2019;37:. [Available online at: https:// ascopubs.org/doi/abs/10.1200/JCO.2019.37.15_suppl.9007; cited 18 April 2020]

54. Koga T, Suda K, Nishino M, et al. Potent in vitro activity of tarloxotinib for HER2 exon 20 mutations in lung cancer and mechanism of acquired resistance [abstract P2.03-20]. J Thorac Oncol 2019;14:S690-1.
55. Haura EB, Cho BC, Lee JS, et al. JNJ-61186372 (JNJ-372), an EGFRcMet bispecific antibody, in EGFR-driven advanced non-small cell lung cancer (NSCLC) [abstract 9009]. JClin Oncol 2009;37: [Available online at: https://ascopubs.org/doi/abs/10.1200/ JCO.2019.37.15_suppl.9009; cited 18 April 2020]

56. Kobayashi S, Boggon TJ, Dayaram T, et al. EGFR mutation and resistance of non-small-cell lung cancer to gefitinib. $N$ Engl J Med 2005;352:786-92.

57. Pao W, Miller VA, Politi KA, et al. Acquired resistance of lung adenocarcinomas to gefitinib or erlotinib is associated with a second mutation in the EGFR kinase domain. PLoS Med 2005;2: 73.

58. Wu SG, Liu YN, Tsai MF, et al. The mechanism of acquired resistance to irreversible EGFR tyrosine kinase inhibitorafatinib in lung adenocarcinoma patients. Oncotarget 2016;7:12404-13.

59. Stockley T, Souza CA, Cheema PK, et al. Evidence-based best practices for EGFR T790M testing in lung cancer in Canada. Curr Oncol 2018;25:163-9.

60. Mok TS, Wu YL, Ahn MJ, et al. Osimertinib or platinumpemetrexed in EGFR T790M-positive lung cancer. $N$ Engl J Med 2017;376:629-40.

61. Wu YL, Mok TSK, Han JY, et al. Overall survival (OS) from the AURA3 phase III study: osimertinib vs platinum-pemetrexed (plt-pem) in patients (pts) with EGFR T790M advanced non-small cell lung cancer (NSCLC) and progression on a prior EGFR-tyrosine kinase inhibitor (TKI) [abstract]. Ann Oncol 2019;30(suppl 9):ix158.

62. Hochmair MJ, Morabito A, Hao D, et al. Sequential treatment with afatinib and osimertinib in patients with EGFR mutation-positive non-small-cell lung cancer: an observational study. Future Oncol 2018;14:2861-74.

63. O'Kane GM, Barnes TA, Leighl NB. Resistance to epidermal growth factor receptor tyrosine kinase inhibitors, T790M, and clinical trials. Curr Oncol 2018;25(suppl 1):S28-37.

64. Socinski MA, Jotte RM, Cappuzzo F, et al. Atezolizumab for first-line treatment of metastatic nonsquamous NSCLC. NEngl JMed 2018;378:2288-301.

65. Reck M, Mok TSK, Nishio M, et al. on behalf of the IMpower150 study group. Atezolizumab plus bevacizumab and chemotherapy in non-small-cell lung cancer (IMpower150): key subgroup analyses of patients with EGFR mutations or baseline liver metastases in a randomised, open-label phase 3 trial. Lancet Respir Med 2019;7:387-401.

66. Cho BC, Cheng Y, Zhou C, et al. Mechanisms of acquired resistance to first-line osimertinib: preliminary data from the phase III FLAURA study [abstract LBA50]. Ann Oncol 2018;29(suppl 8):ix177.

67. Oxnard G, Hu Y, Mileham K, et al. Osimertinib resistance mediated by loss of EGFR T790M is associated with early resistance and competing resistance mechanisms [abstract OA 09.02]. J Thorac Oncol 2017;12:S1767-8.

68. Arulananda S, Do H, Musafer A, Mitchell P, Dobrovic A, John T. Combination osimertinib and gefitinib in C797S and T790M EGFR-mutated non-small cell lung cancer. J Thorac Oncol 2017;12:1728-32.

69. Husain H, Martins R, Goldberg S, et al. Phase 1 dose escalation of PF-06747775 (EGFR-T790M inhibitor) in patients with advanced EGFRm (del 19 or L858R+/-T790M) NSCLC [abstract P3.02b-001]. J Thorac Oncol 2017;12(suppl):S1185.

70. Shi Y, Hu X, Zhang S, et al. Third generation EGFR inhibitor AST2818 (alflutinib) in NSCLC patients with EGFR T790M mutation: a phase $1 / 2$ multi-center clinical trial [abstract P2.03-028]. J Thorac Oncol 2017;12 (suppl 2):S2138.

71. ChoBC,HanJY,KimSW,etal.YH25448, a3rdgenerationEGFR-TKI, in patients with EGFR-TKI-resistant NSCLC: phase I/II study results [abstract 9033]. JClin Oncol 2018;36:. [Available online at: 
https://ascopubs.org/doi/abs/10.1200/JCO.2018.36.15_suppl. 9033; cited 19 April 2020]

72. Xu W, Tang W, Li T, Zhang X, Sun $Y$. Overcoming resistance to AC0010, a third generation of EGFR inhibitor, by targeting c-Met and Bcl-2. Neoplasia 2019;21:41-51.

73. Tan DS, Kim SW, Sequist LV, et al. Phase II results for single-agent nazartinib (EGF816) in adult patients (pts) with treatment-naïve EGFR-mutant non-small cell lung cancer (NSCLC) [abstract LBA61]. Ann Oncol 2018;29(suppl 8):viii748.

74. Oxnard GR, Cantarini M, Frewer P, et al. SAVANNAH: a phase II trial of osimertinib plus savolitinib for patients (pts) with $E G F R$-mutant, $M E T$-driven (MET+), locally advanced or metastatic non-small cell lung cancer (NSCLC), following disease progression on osimertinib [abstract TPS9119]. J Clin Oncol 2019;37:. [Available online at: https://ascopubs.org/doi/ abs/10.1200/JCO.2019.37.15_suppl.TPS9119; cited 19April2020]

75. Hartmaier RJ, Han JY, Cho BC, et al. Detection of METmediated EGFR tyrosine kinase inhibitor (TKI) resistance in advanced non-small cell lung cancer (NSCLC): biomarker analysis of the TATTON study [abstract 4897]. Cancer Res 2019; 79(suppl):S2209.

76. Ahn M, Han J, Sequist L, et al. тATTON ph Iв expansion cohort: osimertinib plus savolitinib for pts with EGFR-mutant $M E T$-amplified NSCLC after progression on prior EGFR-TKI [abstract OA 09.03]. J Thorac Oncol 2017;12:S1768.

77. Yu H, Ahn NJ, Kim SW, et al. TATTON phase Iв expansion cohort: osimertinib plus savolitinib for patients (pts) with EGFRmutant, $M E T$-amplified NSCLC after progression on prior first/ second-generation epidermal growth factor receptor (EGFR) tyrosine kinase inhibitor (TKI) [abstract CT032]. Cancer Res 2019;79(suppl 13):. [Available online at: https://cancerres. aacrjournals.org/content/79/13_Supplement/CT032; cited 30 April 2020]

78. Sequist LV, Lee JS, Han JY, et al. TATTON phase IB expansion cohort: osimertinib plus savolitinib for patients (pts) with $E G F R$-mutant, $M E T$-amplified NSCLC after progression on prior third-generation epidermal growth factor receptor (EGFR) tyrosine kinase inhibitor (TKI) [abstract CT033]. Cancer Res 2019;79(suppl 13):. [Available online at: https://cancerres. aacrjournals.org/content/79/13_Supplement/CT033; cited 30 April 2020]

79. Oxnard GR, Ramalingam SS, Ahn MJ, et al. Preliminary results of TATTON, a multi-arm phase IB trial of AZD9291 combined with MEDI4736, AZD6094 or selumetinib in EGFR-mutant lung cancer [abstract 2509]. J Clin Oncol 2015; 33:. [Available online at: https://ascopubs.org/doi/abs/10. 1200/jco.2015.33.15_suppl.2509; cited 19 April 2020]

80. Ramalingam SS, Saka H, Ahn MJ, et al. Osimertinib plus selumetinib for patients (pts) with EGFR-mutant (EGFRm) NSCLC following disease progression on an EGFR-TKI: results from the phase IB TATTON study [abstract CT034]. Cancer Res 2019;79(suppl 13):. [Available online at: https://cancerres. aacrjournals.org/content/79/13_Supplement/CT034; cited 30 April 2020]

81. Harrison C, Vannucchi AM. Ruxolitinib: a potent and selective Janus kinase 1 and 2 inhibitor in patients with myelofibrosis. An update for clinicians. Ther Adv Hematol 2012;3:341-54.

82. Janne PA, Yu HA, Johnson ML, et al. Safety and preliminary antitumour activity of U3-1402: a HER3-targeted antibody drug conjugate in EGFR TKI-resistant, EGFRm NSCLC [abstract 9010]. J Clin Oncol 2019;37:. [Available online at: https:// ascopubs.org/doi/abs/10.1200/JCO.2019.37.15_suppl.9010; cited 19 April 2020]

83. Nakamura A, Inoue A, Morita S, et al. Phase III study comparing gefitinib monotherapy $(\mathrm{G})$ to combination therapy with gefitinib, carboplatin, and pemetrexed (GCP) for untreated patients (pts) with advanced non-small cell lung cancer (NSCLC) with EGFR mutations (NEJ009) [abstract 9005]. J Clin Oncol 2018;36:. [Available online at: https://ascopubs.org/ doi/10.1200/JCO.2018.36.15_suppl.9005; cited 19 April 2020]

84. Seike M, Inoue A, Sugawara S, et al. Phase III study of gefitinib (G) versus gefitinib+carboplatin+pemetrexed (GCP) as firstline treatment for patients (pts) with advanced non-small cell lung cancer (NSCLC) with EGFR mutations (NEJ009) [abstract 1382PD]. Ann Oncol 2018;29:

85. Noronha V, Patil VM, Joshi A, et al. Gefitinib versus gefitinib plus pemetrexed and carboplatin chemotherapy in EGFRmutated lung cancer. J Clin Oncol 2020;38:124-36.

86. Seto T, Kato T, Nishio M, et al. Erlotinib alone or with bevacizumab as first-line therapy in patients with advanced non-squamous non-small-cell lung cancer harbouring EGFR mutations (JO25567): an open-label, randomised, multicentre, phase 2 study. Lancet Oncol 2014;15:1236-44.

87. Furuya N, Fukuhara T, Saito H, et al. Phase III study comparing bevacizumab plus erlotinib to erlotinib in patients with untreated NSCLCharboring activating EGFR mutations: NEJ026 [abstract 9006].JClin Oncol 2018;36:. [Available online at: https:// ascopubs.org/doi/abs/10.1200/JCO.2018.36.15_suppl. 9006; cited 19 April 2020]

88. Garon EB, Reck M, Paz-Ares L, et al. Treatment rationale and study design for the relay study: a multicenter, randomized, double-blind study of erlotinib with ramucirumab or placebo in patients with epidermal growth factor receptor mutation-positive metastatic non-small-cell lung cancer. Clin Lung Cancer 2017;18:96-9.

89. Yamamoto N, Seto T, Nishio M, et al. Erlotinib plus bevacizumab (ЕB) versus erlotinib alone (E) as first-line treatment for advanced EGFR mutation-positive non-squamous non-small-cell lung cancer (NSCLC): survival follow-up results of JO25567 [abstract 9007]. J Clin Oncol 2018;36:. [Available online at: https://ascopubs.org/doi/abs/10.1200/ JCO.2018.36.15_suppl.9007; cited 19 April 2020] 